\title{
How Different are VA Nursing Home Residents?
}

\author{
David R. Mehr, MD, MS,* Brant E. Fries, PhD, $\ddagger$ and Brent C. Williams, MD, MPH†§
}

Objective: To identify similarities and differences between VA nursing home residents and other nursing home residents.

Design: Comparison of cross-sectional data from three sources.

Participants: Residents of VA nursing homes nationwide in early October $1986(n=10,117)$; participants in the 1985 National Nursing Home Survey (NNHS) $(n=5,243)$; residents assessed in New York State nursing homes in 1988 $(n=94,840)$.

Measures: Age-stratified comparisons were made between the VA and the NNHS for gender, marital status, race, ethnicity, length of stay, activities of daily living (ADL) status, and selected diagnoses and conditions. Additionally, casemix data were compared between the VA and the New York State populations.

Main Results: The population of VA nursing homes is over- whelmingly men ( $96.1 \%$ versus $28.4 \%$ in the NNHS), and $31.2 \%$ of the VA population is under 65 years of age compared with $11.6 \%$ in the NNHS. Young $(<65)$ VA residents are considerably more impaired in ADL than young residents in the NNHS; differences are less pronounced in those over 65 years old. VA case mix is slightly higher than the overall New York State population though the distribution of residents into categories in the Resource Utilization Groups, Version II system is somewhat different.

Conclusions: VA nursing homes contain a substantial distinctive population of seriously impaired residents under 65 years of age. Though differences exist, older VA residents have many similarities to residents of non-VA nursing homes and constitute a functionally impaired population that can provide insights into the status of nursing home residents generally. J Am Geriatr Soc 41:1095-1101, 1993

of these units are located on the same site or in close proximity to VA hospitals. Services vary from facility to facility but are typical of services offered in longterm care settings outside of the VA. Some veterans also receive care in community nursing homes under contract from the VA, but we have not considered that setting in this paper.

While some of the conventional wisdom about VA nursing home residents fits out experience, we have observed that beyond the obvious gender differences, many of the same clinical and health policy issues that apply to other nursing home residents apply to the VA. The substantial number of individuals in VA nursing homes nationwide represents an important potential resource for health services research, especially considering the wealth of data contained in accessible VA data systems. Therefore, it is important to describe in what ways VA residents are and are not typical of other nursing home residents.

We combined two VA data systems to create a longitudinal picture of health services utilization by VA nursing home residents. This provided us with the opportunity to examine comparative characteristics of VA and community nursing home residents. Using selection criteria and descriptive characteristics similar to the National Nursing Home Survey (NNHS), we describe a national cross section of VA nursing homes residents and compare them with the current resident sample of the 1985 NNHS. ${ }^{6,7}$ We also compare case mix, using the Resource Utilization Groups, Version II (RUG-II) system, ${ }^{8}$ for the national VA nursing home population and one state-wide population.

\section{METHODS}

Data sources Residents of VA-owned nursing homes are assessed nationwide by nursing home per- 
sonnel semiannually (around October 1 and April 1) and at nursing home admission for the purpose of classification according to the RUG-II system. Responses are stored in the computerized Patient Assessment File (PAF). From the PAF, we identified all 10,482 individuals who had a VA nursing home assessment during the 7-day period ending October 5, 1986. This cross-sectional sample from the PAF was chosen to resemble most closely the "current resident" sample of the 1985 NNHS. Though we are not assured that all mandated assessments were performed and recorded, we have no reason to believe that any systematic exclusions occurred. Therefore, we expect no bias from such missing data, if they exist.

To obtain further demographic and diagnostic information, as well as information about hospital and nursing home utilization, we linked the PAF records with 3 years (Fiscal Years 1986-88) of data from the VA Patient Treatment File (PTF), which provides information for each VA institutional stay ending during a fiscal year. We excluded $365(3.5 \%)$ residents who had records with severe inconsistencies, such as a nursing home admission in the middle of an acute care hospitalization or no record other than one nursing home assessment during this 3-year period. This left 10,117 individuals who constituted our VA population. For information concerning specific chronic diagnoses, we did a supplementary search of all diagnostic data in PTF records for Fiscal Years 1986-89.

As a comparison group, we used the NNHS, a nationwide survey of nursing homes, their residents, discharged residents, and staff conducted by the $\mathrm{Na}$ tional Center for Health Statistics $(n=5,243) .{ }^{7}$ The NNHS is performed periodically, with the two most recent reported surveys from 1977 and 1985. NNHS sampling follows a stratified two-stage probability design: the first stage sampled facilities, and the second sampled residents within facilities. The reported "current resident ${ }^{\prime \prime}$ data describe a cross-sectional view of nursing home residents, and reported findings are weighted estimates designed to be nationally representative. The 1985 current resident data were collected between August 1985 and January 1986, ${ }^{6,7}$ close to the same time period as the VA data.

Sufficient data for detailed measurement of case mix are not available from the NNHS. Therefore, we substituted the nearest available published data from a single state: RUG-II data from the entire population of New York State's nursing home residents assessed for payment determination and quality assurance between July and December $1988(n=94,840) .{ }^{9}$ Seventy-seven percent of these residents were in skilled nursing facilities, with the remainder in intermediate care facilities.

Data Elements Common to VA Files and NNHS We carefully determined which variables of the VA data and the NNHS are reasonably comparable. We identified as common data elements the following: length of stay (LOS) since admission; marital status; ethnic and racial status; dependency in four Activities of Daily Living (ADL) (toileting, transferring, eating, and mobility); diagnoses of diabetes mellitus, COPD, dementia, and alcoholism; and evidence of behavior problems. The specific diagnoses chosen were chronic diseases that we felt could illuminate the relationship between VA and other nursing homes. Some variations in definition do exist as summarized below (further details available from the authors).

Both the VA and the NNHS share common categories for gender, marital status, ethnic status, and racial status; however, some of this information about our VA residents comes from the PTF. Eight percent of the VA residents had no PTF records from Fiscal Years 1986-89. This indicates the absence of any hospital or nursing home discharge during this period. Ethnic and racial status are unknown in this group. Seventeen percent of residents had no PTF record before their assessment; we consider their marital status unknown.

Length of stay was defined in both the NNHS and VA data sets as the time from the date of the resident's most recent admission to the facility to assessment. There is some variability within both data sets as to whether return from an intercurrent hospitalization is counted as a new admission. In the VA, more than 30 days "absent sick in hospital" results in an administrative discharge. While some facilities surveyed for the NNHS treated hospitalization followed by return to the nursing home as part of a single stay, others did not. ${ }^{10}$

The NNHS defines dependency in an ADL as requiring the "help of special equipment and/or another person." ${ }^{\prime 6}$ The closest match to this in the VA is inability to carry out tasks without human assistance or supervision. Residents who only require supervision would likely be classified as independent by the NNHS; the VA definition of dependency may thus be broader. However, a resident who uses adaptive equipment without human assistance or supervision is defined as independent in the VA, which potentially makes the VA definition of dependency narrower.

For the VA, we considered a diagnosis of dementia, alcoholism, diabetes mellitus, or COPD as present if one of the ICD-9-CM diagnosis codes for that condition appeared at least once during Fiscal Years 1986-1989. For dementia, codes for both dementia and Alzheimer's disease were identified. Though we may have misclassified some residents who truly had the initial onset of dementia, alcoholism, diabetes mellitus, or COPD after October 1986, we deliberately chose a long time period in which to identify these chronic diagnoses to avoid underdiagnosis and missing data. Frequently such chronic conditions are not listed at a single hospital or nursing home discharge. Furthermore, since only the PTF has diagnostic data, using a more restricted period would have meant substantially more residents with no diagnostic information at all (missing data).

For the NNHS, we took information on diabetes mellitus, COPD, and dementia from the current diagnosis section. As with the VA, diagnosed Alzheimer's Disease was also added to the dementia category. Because alcoholism is not present in the diagnosis section of the NNHS, we substituted results from the "current mental disorders" section (based on staff perceptions rather than recorded diagnoses) for this diag- 
nosis. Behavior problems is also a category in the "current mental disorders" section of the NNHS; we used the definition of the RUG-II Behavior Problem category as our standard of comparison for the VA.

RUG-II System The RUG-II system is designed to classify nursing home residents into distinct groups that are descriptive of the cost of their care. Individuals are placed first within a hierarchy of five major categories, which are then divided into a total of $16 \mathrm{mu}$ tually exclusive groups on the basis of an index of ADL dependency. The hierarchy categories include the following:

- Heavy Rehabilitation-physical or occupational therapy at least 30 minutes per day, at least 5 days per week, with a goal of restoration of functioning;

- Special Care-coma, nasogastric feeding, parenteral feeding, quadriplegia, multiple sclerosis, stage 4 decubiti, suctioning;

- Clinically Complex-selected treatments (eg, oxygen therapy, wound/lesion care), diagnoses (eg, hemiplegia), conditions (eg, dehydration, stasis ulcer), frequent physician visits, or Special Care with mild or absent ADL dependency;

- Severe Behavior Problem-physical aggression, verbal abuse, regressive behavior, or hallucinations present at the severe level;

- Reduced Physical Function-residents not classified in the other four categories.

The RUG-II system is described in more detail elsewhere. $^{8}$

Analysis Both the VA and New York State data represent entire populations. In contrast, figures reported for the NNHS represent population point estimates based on weighted data. Statistical tests are technically inappropriate for comparing a population and a sample. We nonetheless computed $95 \%$ confidence intervals from the NNHS data (based on information for computing standard errors in the technical appendices) to see if the VA results fell outside of the confidence bounds. Given the large sample size of the NNHS, it was not surprising that in almost all cases, even with similar results, VA results fell outside the 95\% confidence limits from the NNHS. Therefore, in comparing the different data sets, we do not in general report any tests of statistical inference or whether a specific result fell outside the NNHS $95 \%$ confidence limits. Instead, we suggest that the reader consider whether the magnitude of the difference is likely to be important or not. This is an issue of judgement rather than statistics.

In comparing the case-mix data for the VA and the New York State populations, we use the RUG-II case-mix index. For any sample, this is computed by weighting the proportion of residents in each of the 16 RUG-II groups by the group's relative resource use, or case-mix index. The average resident in the original RUG-II derivation sample had a case-mix index set to 1.0 .

\section{RESULTS}

We compared the population of VA nursing home residents (hereafter VA) with the NNHS population es- timates (hereafter NNHS) on a number of dimensions.

Age and Gender The most obvious difference between the two populations is that only $3.9 \%$ of VA residents are women versus $71.6 \%$ in the NNHS. The age distribution is also different. While $11.6 \%$ in the NNHS are under $65,31.2 \%$ in the VA are under 65 . Table 1 lists selected characteristics for the VA and NNHS stratified by age, with results for men in the NNHS also indicated.

The large group of younger (male) residents in the VA is quite distinctive. They exhibit almost as high a proportion of dependency in each ADL as those 65 years of age or older. In contrast, those under 65 in the NNHS are far less likely to be disabled than their VA counterparts or than those in the NNHS who are 65 years old or more. For example, $61.4 \%$ in the VA under age 65 are dependent in transferring, while only $39.6 \%$ ( $37 \%$ of males) in the NNHS under 65 are dependent in transferring.

Marital Status A substantially higher proportion of residents in the VA are married than are residents in the NNHS; men in the NNHS are intermediate. In the VA, there is a decline in the proportion married from $50.6 \%$ for ages 65 to 74 to $34.1 \%$ for those over 85 (not shown). For men in the NNHS, the proportion married does not vary substantially (between $26 \%$ and $30 \%$ ) for those in age groups over 65 . Women in the NNHS demonstrate a steep decline in the proportion married and a corresponding steep rise in widowhood from ages 65 to 74 to those over 85 (not shown). For men of all ages, the proportion widowed increases with age, more so in the NNHS than in the VA (not shown).

Ethnic and Racial Status The two populations are quite similar in racial distribution, whether comparing all residents or just NNHS males. Hispanic residents are a small minority (under $5 \%$ at each age group). There appears to be a somewhat smaller proportion of residents who are Hispanic in the VA than in the NNHS, but the VA proportion is within the $95 \%$ confidence limits for the NNHS estimate.

Length of Stay Whether looking at males or all residents, the VA has a higher proportion of stays under 3 months. The difference is most marked in those under 65 years old and progressively declines thereafter. For those less than 65 years of age, $24.1 \%$ in the VA versus $14.2 \%$ in the NNHS had an LOS less than 3 months. In contrast, for those over $85,14.1 \%$ in the VA and $11.9 \%$ in the NNHS $(14.8 \%$ for men in the NNHS) had an LOS under 3 months (not shown).

ADL Status ADL dependency in toileting, transferring, and especially eating and mobility are present in a higher proportion of VA residents, although in each case the magnitude of the difference is smaller in those over age 65 . Because in each age group men in the NNHS are less dependent than all residents, if we restrict our comparison to NNHS men, the differences are slightly amplified.

As noted in the methods section, the VA and NNHS definitions for ADL dependency are not exactly equivalent. Overall proportions of dependency in the VA can be checked with reference to the 1990 VA Survey, 
TABLE 1. COMPARISON OF VA RESIDENTS WITH NNHS SAMPLE FOR SELECTED CHARACTERISTICS

\begin{tabular}{|c|c|c|c|c|c|c|c|c|c|}
\hline & \multicolumn{3}{|c|}{ Age Less than 65} & \multicolumn{3}{|c|}{ Age 65 or More } & \multicolumn{3}{|c|}{ All } \\
\hline & \multirow[b]{2}{*}{$\begin{array}{c}\mathbf{V A}^{*} \\
\%\end{array}$} & \multicolumn{2}{|c|}{ NNHS** } & \multirow[b]{2}{*}{$\begin{array}{c}\text { VA } \\
\%\end{array}$} & \multicolumn{2}{|c|}{ NNHS } & \multirow[b]{2}{*}{$\begin{array}{c}\text { VA } \\
\%\end{array}$} & \multicolumn{2}{|c|}{ NNHS } \\
\hline & & $\begin{array}{c}\text { Men } \\
\%\end{array}$ & $\underset{\%}{\text { All }}$ & & $\underset{\%}{\text { Men }}$ & $\underset{\%}{\text { All }}$ & & $\underset{\%}{\operatorname{Men}}$ & $\underset{\%}{\text { All }}$ \\
\hline \multicolumn{10}{|l|}{ Gender } \\
\hline Male & 97.1 & & 51.6 & 95.6 & & 25.4 & 96.1 & & 28.4 \\
\hline Female & 2.9 & & 48.4 & 4.4 & & 74.6 & 3.9 & & 71.6 \\
\hline \multicolumn{10}{|l|}{ Marital Status s*** $^{*}$} \\
\hline Married & 41.1 & 10.8 & 11.5 & 44.5 & 28.1 & 12.8 & 43.4 & 24.5 & 12.6 \\
\hline Widowed & 5.6 & 6.0 & 12.1 & 22.2 & 40.6 & 67.8 & 17.2 & 33.3 & 61.3 \\
\hline Divorced/separated & 28.6 & 24.1 & 22.4 & 14.6 & 10.7 & 5.9 & 18.8 & 13.5 & 7.8 \\
\hline Never married & 22.3 & 59.2 & 54.1 & 16.0 & 20.6 & 13.5 & 17.9 & 28.7 & 18.2 \\
\hline \multicolumn{10}{|l|}{ Race\# } \\
\hline White & 84.3 & 82.7 & 85.1 & 89.3 & 90.7 & 93.1 & 87.8 & 89.0 & 92.2 \\
\hline Black & 14.5 & 15.4 & 12.9 & 9.6 & 8.0 & 6.2 & 11.1 & 9.6 & 7.0 \\
\hline \multicolumn{10}{|l|}{ Ethnicity ${ }^{\#}$} \\
\hline Hispanic & 1.3 & 4.6 & 3.3 & 1.0 & 3.9 & 2.7 & 1.1 & 4.1 & 2.7 \\
\hline \multicolumn{10}{|c|}{$\begin{array}{l}\text { Length of stay at time of } \\
\text { data collection }\end{array}$} \\
\hline$<3$ months & 24.1 & 16.5 & 14.2 & 19.1 & 15.3 & 12.7 & 20.7 & 15.5 & 12.9 \\
\hline 3 to $<6 \mathrm{mos}$ & 12.7 & 14.2 & 12.7 & 11.4 & 8.6 & 9.0 & 11.8 & 9.8 & 9.5 \\
\hline 6 to $<12 \operatorname{mos}$ & 15.0 & 11.7 & 12.1 & 15.7 & 14.4 & 14.3 & 15.5 & 13.8 & 14.1 \\
\hline 1 to $<3$ yrs & 23.9 & 24.7 & 24.0 & 27.7 & 33.6 & 32.5 & 26.5 & 31.7 & 31.5 \\
\hline 3 to $<5$ years & 9.4 & 11.3 & 13.0 & 11.1 & 12.5 & 14.0 & 10.6 & 12.2 & 13.9 \\
\hline$\geq 5$ yrs & 15.0 & 21.6 & 24.0 & 15.0 & 15.7 & 17.3 & 15.0 & 16.9 & 18.1 \\
\hline \multicolumn{10}{|l|}{$\begin{array}{l}\text { Activities of Daily Living } \\
\text { percent dependent }\end{array}$} \\
\hline Toileting & 65.0 & 37.7 & 42.9 & 71.4 & 56.1 & 63.2 & 69.4 & 52.2 & 60.9 \\
\hline Transferring & 61.4 & 37.0 & 39.6 & 67.7 & 55.2 & 62.6 & 65.7 & 51.4 & 59.9 \\
\hline Eating & 68.4 & 29.0 & 31.5 & 70.9 & 34.7 & 40.3 & 70.2 & 33.5 & 39.3 \\
\hline Mobility & 72.1 & & 49.0 & 79.4 & & 73.6 & 77.2 & & 70.7 \\
\hline \multicolumn{10}{|c|}{ Diagnoses and conditions } \\
\hline $\mathrm{COPD}^{\# \#, \# \# \#}$ & 16.8 & 21.9 & 4.0 & 24.4 & 12.8 & 7.2 & 22.1 & 10.6 & 6.8 \\
\hline Diabetes mellitus $\# \# \#$ & 15.9 & 9.9 & 11.7 & 18.3 & 12.4 & 12.5 & 17.5 & 11.9 & 12.4 \\
\hline Dementia $\# \#$ & 9.0 & 12.7 & 11.0 & 23.2 & 27.7 & 30.5 & 18.9 & 25.5 & 28.3 \\
\hline Alcoholism & 12.8 & 13.5 & 8.2 & 6.9 & 5.7 & 2.1 & 8.7 & 7.4 & 2.8 \\
\hline Behavior problem & 34.7 & 38.2 & 39.7 & 33.3 & 38.8 & 38.2 & 33.8 & 38.7 & 38.4 \\
\hline
\end{tabular}

which used an ADL definition identical to that used in the NNHS. ${ }^{5}$ The comparisons for the four ADL dependencies between our overall findings and the 1990 VA Survey, respectively, are as follows: toileting $69.4 \%$ (66\% in the 1990 VA Survey), transferring $65.7 \%$ $(60 \%)$, eating $70.2 \%(60 \%)$, and mobility $77.2 \%(68 \%)$. These figures suggest that the estimates from our VA data are slightly high, with eating dependency the least accurate. Considering the definitions involved, the overestimate of eating dependency in the VA is not surprising. A VA resident requiring supervision is defined as dependent in eating. This is least like assistance of all of the ADLs examined. These estimates from the 1990 VA Survey reinforce similarity in dependency between the VA and the NNHS for those over 65 years of age.

Mental Disorders and Behavior Problems Behavior problems are slightly less prevalent in the VA than in the NNHS population in residents both older and younger than 65. Alcoholism declines with age to levels below $2 \%$ in both populations for those 85 years old and more (not shown), and it is uncommon in either population in those 65 years of age or more. The prevalence of diagnosed alcoholism is higher in the VA at all ages in comparison with the NNHS, but the VA proportions are almost identical to the proportions of men of similar age from the NNHS. Additionally, only $12.8 \%$ of the VA population under age 65 , has had a diagnosis of alcoholism. The VA figure may be an underestimate because it is based on diagnosis. In contrast to the overall VA proportion of alcoholism from our data of $8.7 \%$, the overall proportion of alcoholism from the 1990 VA Survey is about $15 \%$. With the NNHS and the 1990 VA Survey figures for alcoholism based on staff perceptions, the actual magnitude of the problem in either locus could be even higher.

Diagnosed dementia increases with age in both populations, and estimates are very similar, particularly 
when comparing men only from the NNHS. Both figures are probably underestimates of the true prevalence of dementia. The overall prevalence of "senile dementia or chronic organic brain syndrome" from the current mental disorders section of the NNHS, where staff report their perceptions irrespective of medical record diagnosis, is $43.4 \%$ compared with $28.3 \%$ from the diagnosis section. The comparable figure to the NNHS current mental disorders estimate from the 1990 VA Survey is about $39 \%$, with about $20 \%$ overall having a primary diagnosis of dementia.

Other Diagnoses COPD was diagnosed at some time between October 1985 and October 1989 in 22\% of the VA nursing home residents. In those under age $65,21.9 \%$ of the men in the NNHS and $16.8 \%$ of individuals in the VA had this diagnosis. In contrast, for those over age 65, COPD was more prevalent in the VA $(24.4 \%)$ residents than in men in the NNHS $(12.8 \%)$. Diabetes mellitus is somewhat more common in the VA than in the NNHS.

RUG-II Distribution Table 2 compares the RUGII distribution for the VA and New York State both in designated skilled nursing facilities (SNFs) and for all residents. The Heavy Rehabilitation and Special Care hierarchy categories are larger in the VA and especially prevalent for those under 65 . In contrast, there are many fewer VA residents in the Reduced Physical Function category, and this is particularly true in the under 65 age group. This difference is principally because of many fewer VA residents with moderately severe ADL impairment who do not have characteristics placing them in more resource-intense categories (not shown).

The average case-mix index for all VA residents (1.07) is higher than for all New York State residents (1.04), but lower than for New York State SNF residents (1.16). VA residents under 65 require substantially more care on average than older VA residents, but despite a somewhat different distribution, VA residents over 65 have overall care requirements very similar to all New York State nursing home residents.

\section{DISCUSSION}

Several observations emerge from the comparison of selected characteristics of VA and other nursing home residents. Beyond the obvious gender difference, the large group of young (under 65), physically dependent residents in the VA is unique. They are quite impaired with much higher dependency in toileting, transferring, eating, and mobility than those under 65 years of age in non-VA nursing homes. The young VA residents have a high case mix and are relatively unlikely to be in the RUG-II Reduced Physical Function category, the default category.

We might speculate that this large group comprises at least in part veterans with chronic service-related injuries. In support of this, quadriplegia is much more prevalent in the VA in those under 65 years of age $\left(9.3 \%\right.$ vs $2.3 \%$ for those 65 years old or more; $\chi^{2}=$ 246.95, $P<0.00001$ ), and hemiplegia is slightly more prevalent $\left(24.3 \%\right.$ vs $21.6 \%$ respectively; $\chi^{2}=8.62$, $P=0.003$ ). Younger residents in non-VA nursing homes have a high prevalence of developmental disability and mental disorders. Seventy-eight percent of those under 65 in the NNHS have one or more mental disorders, including mental retardation $(26 \%)$, dementia (16\%), depressive disorders (16\%), and schizophrenia $(24 \%)$. Comparable figures for hemiplegia or quadriplegia are not available in the NNHS, nor are comparable figures available for most of the above mental disorders in the VA.

In those individuals 65 years of age and older, there are also differences between VA and NNHS residents, but they are often less significant. This particularly holds true for length of stay and ADL dependencies. Racial and ethnic status, mental disorders, and the prevalence of diabetes are similar between the VA and the NNHS in both older and younger residents. Diagnosed alcoholism is not appreciably more common in the VA than in men in the NNHS and in both data sets declines markedly with age. Though the 1990 VA Survey suggests that our data somewhat underestimate the prevalence of alcoholism in the VA, the trend toward decline with aging is probably real. Marital status and diagnosed COPD are the areas of greatest divergence.

Using the RUG-II system, we see that VA facilities have a higher proportion of residents in heavier care categories, especially among residents under 65 years of age. In addition to the high prevalence of nervous system disorders noted above, rehabilitation in the VA

TABLE 2. RUG-II DISTRIBUTION AND AVERAGE CASE MIX IN VA AND NEW YORK STATE NURSING HOMES*

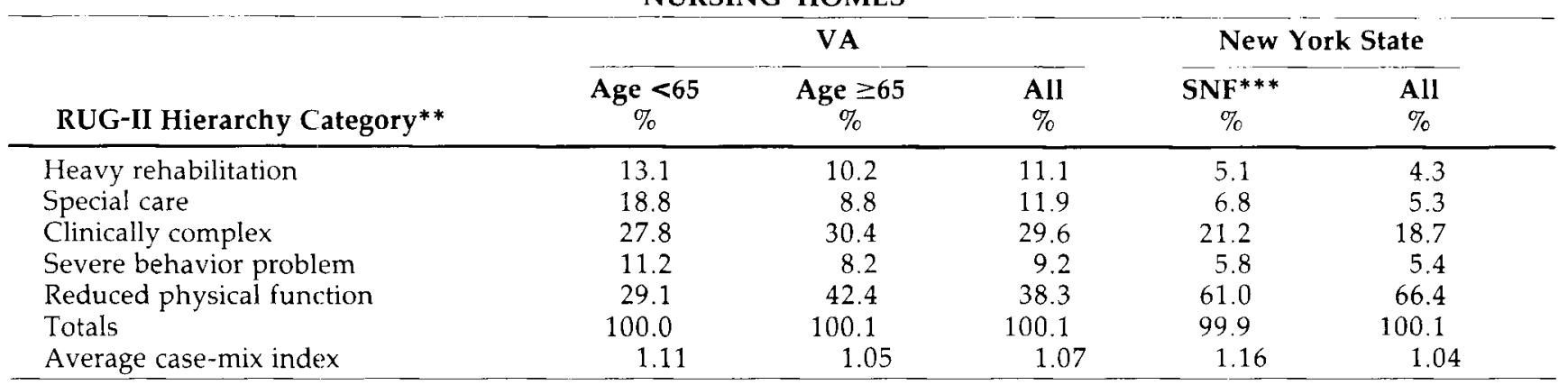

* Totals may not add to $100 \%$ due to rounding.

** See methods for definitions. Further information in Schneider et al, 1988. ${ }^{10}$

*** Skilled Nursing Facility. 
operates under different rules than in nursing homes outside the VA system. In non-VA nursing homes, Medicare skilled care is the usual mechanism for funding rehabilitation. Whereas Medicare reimbursement for skilled care is limited to 100 days, ${ }^{11}$ VA residents may continue in rehabilitation programs if appearing to make progress for substantially longer periods. In our experience, long-term rehabilitation is far more common in the VA nursing home system. Outcomes of rehabilitation in VA nursing homes may be a worthwhile subject for further study.

The finding of shorter stays in the VA is contrary to popular perceptions and distinct from findings for hospital care. ${ }^{12}$ Inconsistency in the definition of length of stay within the NNHS biases reported NNHS estimates in the direction of shorter stays. Therefore our finding of shorter stays in the VA would be even more prominent with more accurate data from the NNHS. In another publication, we have examined predictors of length of stay for VA nursing home residents admitted during the first half of Fiscal Year $1987 .{ }^{13}$ In that study, $72 \%$ of both brief- ( $<1$ month) and medium- (1-6 month) stay residents were discharged alive. Brief-stay residents, when compared with medium-stay and longstay residents, respectively, were more likely to be terminally ill (17\% vs $9 \%$ and $4 \%$ ) and were more likely to have had a prior VA nursing home stay $(24 \%$ vs $6 \%$ and $9 \%$ ). All three groups had a high proportion of at least one ADL dependency $(56 \%$ vs $51 \%$ and $62 \%$ ).

There are several limitations to our study. First, data collection for the 1985 NNHS is not very distant from October 1986, but the New York State data is 2 years later. The overall case-mix index has increased over time in New York State, but the increase was quite small between the fourth quarter of 1986, and the data we report from 1988 . The time difference in data should accentuate the slightly higher case-mix index of VA nursing home residents but not to the extent that VA nursing homes provide the same intensity of care as strictly skilled facilities. As VA nursing homes are heterogeneous, this result does not surprise us.

Second, some of the comparisons between the NNHS and the VA do not apply precisely the same definition. Given differences in data collection instruments, we attempted to make the closest match possible. The areas of greatest potential divergence are the ADLs and the diagnosis of alcoholism.

The 1990 Survey of VA Nursing Homes puts our definitional choices in perspective. Although only overall results for the VA system have been reported, the survey instrument used definitions virtually identical to the NNHS. Compared with the 1990 VA Survey, our data identify slightly higher overall prevalences of ADL dependencies, with eating dependency the most different. We also identify somewhat less alcoholism. The overestimation of eating dependency in our data suggests that for those over age 65, ADL dependency in the VA and in non-VA nursing homes are even more similar than the data presented in Table 1. As with the NNHS, the 1990 VA Survey demonstrates that diagnosed dementia is much less common than dementia defined by caregivers; however, this does not invalidate our comparison of diagnosed dementia in the VA and the NNHS. The 1990 VA Survey figure for the overall prevalence of dementia is quite close to the NNHS overall estimate, although the age distributions are different.

A third limitation is the absence of several important social variables in the VA data. We can, however, estimate some relationships. Users of VA care overall are known to be of lower socioeconomic status than the overall US population ${ }^{3,4}$; therefore one might expect the same of the VA nursing home population. We have no exact standard of comparison, but poverty is also common in community nursing home care, with $50.4 \%$ in the NNHS on Medicaid in the month before the survey. ${ }^{7}$ The higher percentage of married veterans may indicate the availability of more social support, which could contribute to the observed shorter average length of stay. Conversely, the smaller proportion of married men in non-VA nursing homes might reflect financial disincentives for the spouse. Until new federal regulations took effect in September 1989, gaining Medicaid benefits for a married nursing home resident in many states required the virtual impoverishment of the non-institutionalized spouse. ${ }^{14}$ In either case, understanding the difference between these two populations in marital status might provide important clues as to whether overutilization of institutional care is occurring in the VA or underutilization in the community. This is an area requiring further study.

Finally, we have no way of comparing the process of care inside and outside the VA. We know from the comparison with the New York State data that a higher proportion of VA residents receive active rehabilitation programs. Though we believe that rehabilitation is provided differently in the VA, the difference could also be due to different resources or different patients.

We considered to what extent differences between the VA and the NNHS in age distribution could affect our results. Of those VA nursing home residents at least age 65 , the proportions in the 65-74, 75-84, and $85+$ subgroups are $50.1 \%, 25.0 \%$, and $24.9 \%$, respectively. The corresponding figures for the NNHS overall are $16.1 \%, 38.6 \%$, and $45.3 \%$ (for men only $24.1 \%$, $42.2 \%$, and 33.7). Because the VA has a smaller proportion of very old individuals, some findings considering all residents over age 65 could be biased by the different age distributions. Since there are very few women in the VA population, we felt it would be inappropriate to inflate greatly their impact by weighting to the same age-sex distribution as the NNHS. Therefore, we applied a simple direct age adjustment to men 65 years old or older in the VA population, using as the standard the NNHS age distribution of men over 65 years old. This proved to have almost no impact, changing our estimates in almost all cases less than $1 \%$. The biggest difference was for the diagnosis of dementia, which after age adjustment increased from $23.2 \%$ to $25.5 \%$.

Though the number of residents in long-term care in the VA is dwarfed by the rest of the long-term care system (NNHS population estimate is $1,491,400$ ), the 
VA is a potentially important resource for biomedical, social, and health services research. Specifically concerning the last of these, the VA has important advantages that at present exist nowhere else: (1) the VA has collected computerized data with consistent definitions about hospital and nursing home utilization and nursing home assessments for a number of years; (2) though we have no definitive data, we expect that veterans using VA services will receive a substantial proportion of their care from the VA; and (3) for those veterans who qualify, the VA provides a model of a health care system without access costs.

Some have argued that the VA is so distinctive in population and in organization that no useful conclusions can be drawn on the basis of VA experience. Despite its limitations, our study does demonstrate that within the VA system, there is a heavy care population of elderly residents who have severe functional disabilities similar to elderly nursing home residents represented in the NNHS. We believe that this provides a starting place for a variety of types of studies of VA nursing home residents that have the potential for some generalizability outside of the VA. This is particularly true if comparisons are limited to men, and residents under age 65 are treated separately.

Studies of clinical conditions, natural history of disease, and clinical interventions are clearly appropriate with the caveat that the interpretation of all studies needs to account for the composition of the study population. Additionally, though not fully comparable and operating with somewhat different system constraints, studies of the outcome of nursing home care and of patterns of institutional utilization by nursing home residents may provide insights concerning the clinical course and health services utilization of an impaired population of community nursing home residents. As this kind of data becomes more available outside of the VA system, fuller comparison will be possible.

\section{ACKNOWLEDGMENTS}

This study would not have been possible without the assistance of Elizabeth Bates, PhD and her staff at the Great Lakes Field Office of the VA Health Services Research and Development Program. Chris Young provided programming assistance. Carol Lynn Dacko assisted with preparation of tables. A. Regula Herzog, $\mathrm{PhD}$ of the University of Michigan, Laurence Z. Rubenstein, MD, MPH, Director of the Sepulveda VA GRECC, Robert Blake, Jr., MD, of the University of Missouri, and two anonymous reviewers provided helpful comments concerning earlier versions of this manuscript. Karen Davenport assisted in manuscript preparation.

\section{REFERENCES}

1. Kosloski K, Austin C, Borgatta E. Determinants of VA utilization. The 1983 survey of aging veterans. Med Care 1987;25:830-846.

2. Wolinsky FD, Coe RM, Mosely RR $2 d$ et al. Veterans' and nonveterans' use of health services. A comparative analysis. Med Care 1985;23:13581371.

3. Randall $M$, Kilpatrick KE, Pendergast JF et al. Differences in patient characteristics between Veterans Administration and community hospitals. Implications for VA planning. Med Care 1987;25:1099-1104.

4. Wolinsky FD, Coe RM, Mosely RR 2d et al. Some clarification about health planning in the VA. Med Care 1987;25:1105-1110.

5. Office of Geriatrics and Extended Care, Office of Finance and Planning. 1990 survey of VA nursing homes. Department of Veterans Affairs working document. September 17, 1990.

6. Hing E, Sekscenski E, Strahan G. The National Nursing Home Survey: 1985 summary for the United States. Washington, DC: National Center for Health Statistics. Vital Health Stat 1989 (97).

7. Hing E. Nursing home utilization by current residents: United States, 1985 Washington, DC: National Center for Health Statistics. Vital Health Stat 1989;13(102).

8. Schneider DP, Fries BE, Foley WJ et al. Case mix for nursing home payment: Resource utilization groups, version II. Health Care Financ Rev 1988; Suppl:39-52.

9. Fries BE, Ljunggren $G$, Winblad B. International comparison of long-term care: The need for resident-level classification. J Am Geriatr Soc 1991;39:10-16

10. Jonas BS, Madans JH, Rothwell ST et al. A method to redefine stays on the 1985 National Nursing Home Survey. Vital Health Stat 1992;2(115).

11. Prospective Payment Assessment Commission. Medicare's skilled nursing facility payment reform. Congressional Report C-92-01. Washington, DC: U.S. Government Printing Office, 1992.

12. Rogers JL, Feinglass J, Martin GJ et al. Longer hospitalization at Veterans Administration hospitals than private hospitals. Verification and additional insights. Med Care 1989;27:928-936.

13. Williams BC, Fries BE, Mehr DR. Length of stay in VA nursing homes: Comparative characteristics of brief-, medium-, and long-stay residents. J Aging Health 1993;5:208-228.

14. 42 U.S.C. $\$ \S 1396 \mathrm{r}-5$ (1988) 